\title{
I CONGRESO INTERNACIONAL DE AVANCES EN SALUD HUMANA "CONSTRUCCIÓN DE UNA NUEVA AGENDA". UNIVERSIDAD NACIONAL DE VILLA MARÍA, CÓRDOBA, ARGENTINA, 25 Y 26 DE OCTUBRE DE 2018
}

Una nutrida participación de profesores extranjeros y otros tantos nacionales brindaron el marco propicio para que se cumpliera con todo éxito el I Congreso Internacional de Avances en Salud Humana y que llevaba por lema: "Construcción de una nueva agenda". Las actividades se cumplieron en la Ciudad de Villa María, localidad a unos 150 Km. de la Ciudad de Córdoba, Argentina, y donde hace cuatro años se inauguró la primera cohorte en la carrera de grado de Medicina, que se suma a otras carreras vinculadas al sector salud ofrecidas en la Universidad Nacional de Villa María; casa académica que está pronta a cumplir sus iniciales 25 años de vida institucional.

El Primer Congreso Internacional de Avances en Salud Humana fue organizado por el Instituto Académico Pedagógico de Ciencias Humanas (IAPCH) de la mencionada universidad, desde sus carreras de Medicina, Terapia Ocupacional, Enfermería y el Centro de Ciencias de la Salud "Bernardo Houssay". Se aspiró consolidar, a lo largo de los desarrollos expositivos y discusiones consecuentes, el enfoque humanista, interdisciplinar e intersectorial como eje transversal en la promoción de la actualización científica, en el debate sobre avances en el área de la salud humana, como así también en la construcción de una agenda de prioridades sobre retos actuales y futuros de la salud de la población, a nivel regional y global.

La propuesta se cumplió sobre la base de los siguientes ejes: salud pública, clínico quirúrgico y comunitario. En breve anotación se puede apuntar, para el primero de ellos, temáticas referidas a promoción de la salud y prevención de enfermedades y daños, en relación a la protección de la población respecto de enfermedades no trasmisibles (ENT), específicamente referidas a las últimas investigaciones sobre cuidados a la mujer con enfermedad coronaria; sobre los enfoques actuales y programas en VIH y en sus implementaciones; respecto de enfoques de políticas públicas, nacionales y locales, acerca del tema del consumo problemático de drogas, junto a la propuesta de prácticas de cuidado desde una perspectiva integral, comunitaria e interdisciplinaria.

El segundo de los ejes tuvo como núcleo de abordaje los nuevos desafíos en el tratamiento y el cuidado de la personas con dolor agudo y crónico, farmacovigilancia, avances de la rehabilitación con la tecnología y el trabajo interdisciplinario, diagnóstico diferencial de enfermedades poco frecuentes y avances en el diagnóstico de cánceres de mayor frecuencia. Además, propuestas de cuidados paliativos a personas que transitan diferentes experiencias de salud, limitantes para la vida, o en situación de final de la vida, desde un enfoque psicosocial e interdisciplinario. Se abordó la bioética en salud en cuestiones que tensionan los avances tecnológicos y el encarnizamiento terapéutico, muerte digna y legislación sobre derechos de los pacientes. El último de los ejes incluyó disertaciones abiertas a la comunidad general, cuyo objetivo fue acercar a los habitantes de Villa María a diversas temáticas relacionadas con la salud en la infancia y en la adolescencia.

Asistieron al mencionado Congreso Internacional aproximadamente 500 profesionales y estudiantes. Se constituyó además como un espacio de encuentro y de socialización de 100 trabajos libres de distintos tipos, tales como trabajos de investigación científica, experiencias en la comunidad, relatos de casos clínicos. Las actividades propuestas brindaron un espacio de reflexión sobre la complejidad de situaciones de cuidado ligadas a las experiencias de vida y salud de las personas, familias, grupos y comunidades, en diversos entornos y etapas evolutivas; permitieron actualizar conocimientos para en- 
frentar, éticamente, esas experiencias de vida y salud, como así también analizar situaciones inherentes a la práctica profesional y al fortalecimiento del juicio crítico sobre temáticas de la salud en general.

Además de reconocidos profesionales de la provincia de Córdoba y de otras provincias de la República Argentina, se sumaron expositores extranjeros de Brasil, Chile, Colombia e Italia. Dentro de los profesores extranjeros, se destacaron los doctores Fernando Lolas Stepke (Chile), Susana Gavalli (Italia), Andrea Donatti Gallassi (Brasil), Maria Alayde Mendonça da Silva (Brasil) y la magíster Fanny Rincón Osorio (Colombia).

El Congreso fue inaugurado con las palabras del señor Intendente de la Ciudad de Villa María, Dr. Martín Gill, y el propio rector de la Universidad Nacional de Villa María, Dr. Luis Negretti, tuvo a su cargo la conferencia de apertura. Por su parte, la decana del Instituto Académico Pedagógico de Ciencias Humanas, Dra. Gloria Vadori, dio inicio al Congreso y tuvo a su cargo el cierre del evento y las conclusiones finales y proyecciones.

Dra. Paula María Cooke

Directora Centro de Ciencias de la Salud "Bernardo Houssay"

I.A.P.C.H.- U.N.V.M. 